Pacific

Journal of

Mathematics

\title{
CONSTRUCTIONS OF PERIODIC MINIMAL SURFACES AND MINIMAL ANNULI IN SOL 3
}

CHRISTOPHE DESMONTS 


\title{
CONSTRUCTIONS OF PERIODIC MINIMAL SURFACES AND MINIMAL ANNULI IN SOL 3
}

\author{
Christophe Desmonts
}

\begin{abstract}
We construct two one-parameter families of minimal properly embedded surfaces in the Lie group $\mathrm{Sol}_{3}$ using a Weierstrass-type representation. These surfaces are not invariant by a one-parameter group of ambient isometries. The first one can be viewed as a family of helicoids, and the second one as a family of minimal annuli called catenoids. Finally we study limits of these catenoids, and in particular we show that one of these limits is a new minimal entire graph.
\end{abstract}

\section{Introduction}

The aim of this paper is to construct two one-parameter families of examples of properly embedded minimal surfaces in the Lie group $\mathrm{Sol}_{3}$, endowed with its standard metric. This Lie group is a homogeneous Riemannian manifold with a 3-dimensional isometry group and is one of the eight Thurston geometries. There is no rotation in $\mathrm{Sol}_{3}$, and so no surface of revolution.

The Hopf differential, which exists on surfaces in every 3-dimensional space form, has been generalized by Abresch and Rosenberg [2004; 2005] to every 3-dimensional homogeneous Riemannian manifold with 4-dimensional isometry group. This tool leads to a lot of works in the field of constant mean curvature $(\mathrm{CMC})$ surfaces in $\mathrm{Nil}_{3}, \widetilde{P S L}_{2}(\mathbb{R})$ and in the Berger spheres. More precisely, Abresch and Rosenberg [2005] proved that the generalized Hopf differential exists in a simply connected Riemannian 3-manifold if and only if its isometry group has at least dimension 4.

Berdinskii and Taimanov [2005] gave a representation formula for minimal surfaces in 3-dimensional Lie groups in terms of spinors, but they pointed out some difficulties for using this theory in the case of $\mathrm{Sol}_{3}$. Nevertheless, some explicit simple examples of minimal surfaces in $\mathrm{Sol}_{3}$ have been constructed in the past decade. Masaltsev [2006] and Daniel and Mira [2013] gave some basic examples of minimal graphs in $\mathrm{Sol}_{3}: x_{1}=a x_{2}+b, x_{1}=a e^{-x_{3}}, x_{1}=a x_{2} e^{-x_{3}}$ and $x_{1}=x_{2} e^{-2 x_{3}}$ (and their images by ambient isometries). López and Munteanu

Keywords: periodic minimal surfaces, minimal annuli, $\mathrm{Sol}_{3}$. 
[2011; 2012; 2014], López [2014] and Masaltsev [2006] studied minimal surfaces in $\mathrm{Sol}_{3}$ invariant by a one-parameter group of ambient isometries. Finally, Ana Menezes [2014] constructed singly and doubly periodic Scherk minimal surfaces in $\mathrm{Nil}_{3}$ and $\mathrm{Sol}_{3}$, and Minh Hoang Nguyen [2014] gave conditions for the Dirichlet problem for the minimal surface equation in $\mathrm{Sol}_{3}$ to have solutions.

The method that we use in this paper is the one used by Daniel and Hauswirth [2009] in $\mathrm{Nil}_{3}$ to construct minimal embedded annuli: We first construct a oneparameter family of embedded minimal surfaces called helicoids and we calculate its Gauss map $g$. A result of Inoguchi and Lee [2008] shows that this map is harmonic for a certain metric on $\overline{\mathbb{C}}$. Then we seek another family of maps $g$ with separated variables that still satisfies the harmonic map equation, and we use a Weierstrass-type representation given by Inoguchi and Lee to construct a minimal immersion whose Gauss map is $g$. We prove that these immersions are periodic, so we get minimal annuli. As far as the authors know, these annuli are the first examples of nonsimply connected minimal surfaces with finite topology (that is, diffeomorphic to a compact surface without a finite number of points) in $\mathrm{Sol}_{3}$.

The model we use for $\mathrm{Sol}_{3}$ is described in Section 2. In the third section, we give some properties of the Gauss map of a conformal minimal immersion in $\mathrm{Sol}_{3}$ (see [Daniel and Mira 2013]). In the fourth section, we construct the family

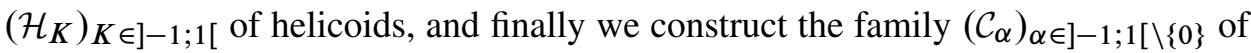
embedded minimal annuli. The study of the limit case of the parameter of this family gives another example of a minimal surface in $\mathrm{Sol}_{3}$, which is an entire graph. None of these surfaces is invariant by a one-parameter family of isometries.

Theorem. There exists a one-parameter family $\left(\mathcal{C}_{\alpha}\right)_{\alpha \in]-1 ; 1[\backslash\{0\}}$ of properly embedded minimal annuli in $\mathrm{Sol}_{3}$, called catenoids, having the following properties:

(1) The intersection of $\mathcal{C}_{\alpha}$ with any plane $\left\{x_{3}=\lambda\right\}$ is a nonempty closed embedded convex curve.

(2) The annulus $\mathcal{C}_{\alpha}$ is conformally equivalent to $\mathbb{C} \backslash\{0\}$.

(3) The annulus $\mathcal{C}_{\alpha}$ has three symmetries fixing the origin: rotation by $\pi$ around the $x_{3}$-axis, reflection in $\left\{x_{1}=0\right\}$ and reflection in $\left\{x_{2}=0\right\}$.

\section{The Lie group $\mathrm{Sol}_{3}$}

Definition. The Lie group $\mathrm{Sol}_{3}$ is $\mathbb{R}^{3}$ with the multiplication $*$ defined by

$$
\left(x_{1}, x_{2}, x_{3}\right) *\left(y_{1}, y_{2}, y_{3}\right)=\left(y_{1} e^{-x_{3}}+x_{1}, y_{2} e^{x_{3}}+x_{2}, x_{3}+y_{3}\right)
$$

for all $\left(x_{1}, x_{2}, x_{3}\right),\left(y_{1}, y_{2}, y_{3}\right) \in \mathbb{R}^{3}$. The identity element is 0 and the inverse element of $\left(x_{1}, x_{2}, x_{3}\right)$ is $\left(x_{1}, x_{2}, x_{3}\right)^{-1}=\left(-x_{1} e^{x_{3}},-x_{2} e^{-x_{3}},-x_{3}\right)$. The Lie group is noncommutative. 
The left multiplication $l_{a}$ by an element $a=\left(a_{1}, a_{2}, a_{3}\right) \in \mathbb{R}^{3}$ is given for all $x=\left(x_{1}, x_{2}, x_{3}\right) \in \mathbb{R}^{3}$ by

$$
\begin{aligned}
l_{a}(x)=a * x & =\left(x_{1} e^{-a_{3}}+a_{1}, x_{2} e^{a_{3}}+a_{2}, a_{3}+x_{3}\right) \\
& =a+M_{a} x,
\end{aligned}
$$

where

$$
M_{a}=\left(\begin{array}{ccc}
e^{-a_{3}} & 0 & 0 \\
0 & e^{a_{3}} & 0 \\
0 & 0 & 1
\end{array}\right)
$$

For the metric $(\cdot, \cdot)$ on $\mathrm{Sol}_{3}$ to be left-invariant, it has to satisfy

$$
\left(M_{a} X, M_{a} Y\right)_{a * x}=(X, Y)_{x}
$$

for all $a, x, X, Y \in \mathbb{R}^{3}$. We define a left-invariant Riemannian metric for $x, X, Y \in \mathbb{R}^{3}$ by the formula

$$
(X, Y)_{x}=\left\langle M_{x^{-1}} X, M_{x^{-1}} Y\right\rangle,
$$

where $\langle\cdot, \cdot\rangle$ is the canonical scalar product on $\mathbb{R}^{3}$ and $x^{-1}$ is the inverse element of $x$ in $\mathrm{Sol}_{3}$. The formula (1) leads to the expression of the previous metric

$$
d s_{x}^{2}=e^{2 x_{3}} d x_{1}^{2}+e^{-2 x_{3}} d x_{2}^{2}+d x_{3}^{2},
$$

where $\left(x_{1}, x_{2}, x_{3}\right)$ are canonical coordinates of $\mathbb{R}^{3}$. Since the translations are isometries now, $\mathrm{Sol}_{3}$ is a homogeneous manifold with this metric.

Remark. This metric is not the only possible left-invariant one on $\mathrm{Sol}_{3}$. In fact, there exists a two-parameter family of nonisometric left-invariant metrics on $\mathrm{Sol}_{3}$. One of these parameters is a homothetic one. The metrics that are nonhomothetic to (2) have no reflections; see [Meeks and Pérez 2012].

By setting

$$
E_{1}(x)=e^{-x_{3}} \partial_{1}, \quad E_{2}(x)=e^{x_{3}} \partial_{2}, \quad \text { and } \quad E_{3}(x)=\partial_{3},
$$

we obtain a left-invariant orthonormal frame $\left(E_{1}, E_{2}, E_{3}\right)$. Thus, we now have two frames to express the coordinates of a vector field on $\mathrm{Sol}_{3}$; we will use brackets to denote the coordinates in the frame $\left(E_{1}, E_{2}, E_{3}\right)$; then at a point $x \in \mathrm{Sol}_{3}$, we have

$$
a_{1} \partial_{1}+a_{2} \partial_{2}+a_{3} \partial_{3}=\left(\begin{array}{l}
a_{1} \\
a_{2} \\
a_{3}
\end{array}\right)=\left[\begin{array}{c}
e^{x_{3}} a_{1} \\
e^{-x_{3}} a_{2} \\
a_{3}
\end{array}\right] .
$$

The following property holds (see [Daniel and Mira 2013]): 
Proposition 1. The isotropy group of the origin of $\mathrm{Sol}_{3}$ is isomorphic to the dihedral group $\mathcal{D}_{4}$ and generated by orientation-reversing isometries

$$
\sigma:\left(x_{1}, x_{2}, x_{3}\right) \longmapsto\left(x_{2},-x_{1},-x_{3}\right) \quad \text { and } \quad \tau:\left(x_{1}, x_{2}, x_{3}\right) \longmapsto\left(-x_{1}, x_{2}, x_{3}\right) \text {, }
$$

whose orders are 4 and 2, respectively.

While $\tau$ is simply a reflection in the plane $\left\{x_{1}=0\right\}$, the generator $\sigma$ can be described as a rotation by $-\pi / 3$ around $E_{3}$ composed with reflection in $\left\{x_{3}=0\right\}$. The cyclic group $\langle\sigma\rangle$ also contains $\sigma^{3}=\sigma^{-1}$ and $\sigma^{2}$, the reflection in $E_{3}$ (rotation by $\pi$ around $E_{3}$ ). The remaining nonidentity elements of the isotropy group of the origin are $\sigma \tau$ and $\sigma^{3} \tau$, which are respectively the reflections in the lines $\left\{\left(x_{1}, x_{1}, 0\right)\right\}$ and $\left\{\left(x_{1},-x_{1}, 0\right)\right\}$, and $\sigma^{2} \tau$, which is reflection in the plane $\left\{x_{2}=0\right\}$.

We deduce the following theorem:

Theorem 2. The isometry group of $\mathrm{Sol}_{3}$ has dimension 3.

Finally, we express the Levi-Civita connection $\nabla$ of $\mathrm{Sol}_{3}$ associated to the metric given by (2) in the frame $\left(E_{1}, E_{2}, E_{3}\right)$. First, we calculate the Lie brackets of the vectors of the frame. The Lie bracket in the Lie algebra $\mathfrak{s o l}_{3}$ of $\mathrm{Sol}_{3}$ is given by

$$
[X, Y]=\left(Y_{3} X_{1}-X_{3} Y_{1}, X_{3} Y_{2}-Y_{3} X_{2}, 0\right)
$$

for all $X=\left(X_{1}, X_{2}, X_{3}\right)$ and $Y=\left(Y_{1}, Y_{2}, Y_{3}\right)$. Then we have

$$
\left[E_{1}, E_{2}\right]=0, \quad\left[E_{1}, E_{3}\right]=E_{1}, \quad\left[E_{2}, E_{3}\right]=-E_{2} .
$$

Hence,

$$
\begin{array}{lll}
\nabla_{E_{1}} E_{1}=-E_{3}, & \nabla_{E_{2}} E_{1}=0, & \nabla_{E_{3}} E_{1}=0, \\
\nabla_{E_{1}} E_{2}=0, & \nabla_{E_{2}} E_{2}=E_{3}, & \nabla_{E_{3}} E_{2}=0, \\
\nabla_{E_{1}} E_{3}=E_{1}, & \nabla_{E_{2}} E_{3}=-E_{2}, & \nabla_{E_{3}} E_{3}=0 .
\end{array}
$$

\section{The Gauss map}

Let $\Sigma$ be a Riemann surface and $z=u+i v$ local complex coordinates in $\Sigma$. Let $x: \Sigma \rightarrow \mathrm{Sol}_{3}$ be a conformal immersion. We set

$$
x=\left(\begin{array}{l}
x_{1} \\
x_{2} \\
x_{3}
\end{array}\right),
$$

and we define $\lambda \in \mathbb{R}_{+}^{*}$ by

$$
2\left(x_{z}, x_{\bar{z}}\right)=\left\|x_{u}\right\|^{2}=\left\|x_{v}\right\|^{2}=\lambda .
$$


Thus, a unit normal vector field is $N: \Sigma \rightarrow T \mathrm{Sol}_{3}$ defined by

$$
N=-\frac{2 i}{\lambda} x_{z} \wedge x_{\bar{z}}:=\left[\begin{array}{l}
N_{1} \\
N_{2} \\
N_{3}
\end{array}\right] \text {. }
$$

Hence we define $\widehat{N}: \Sigma \rightarrow \mathbb{S}^{2} \subset \mathbb{R}^{3}$ by the formula $M_{x^{-1}} N=\widehat{N}$, that is,

$$
\widehat{N}=\left(\begin{array}{ccc}
e^{x_{3}} & 0 & 0 \\
0 & e^{-x_{3}} & 0 \\
0 & 0 & 1
\end{array}\right)\left(\begin{array}{c}
N_{1} e^{-x_{3}} \\
N_{2} e^{x_{3}} \\
N_{3}
\end{array}\right)=\left(\begin{array}{l}
N_{1} \\
N_{2} \\
N_{3}
\end{array}\right) .
$$

Definition. The Gauss map of the immersion $x$ is the application

$$
g=\sigma \circ \hat{N}: \Sigma \longrightarrow \mathbb{C} \cup\{\infty\}=\overline{\mathbb{C}},
$$

where $\sigma$ is the stereographic projection with respect to the southern pole, i.e.,

$$
\begin{aligned}
& N=\frac{1}{1+|g|^{2}}\left[\begin{array}{c}
2 \Re(g) \\
2 \Im(g) \\
1-|g|^{2}
\end{array}\right], \\
& g=\frac{N_{1}+i N_{2}}{1+N_{3}}
\end{aligned}
$$

The following result is due to [Inoguchi and Lee 2008]. It can be viewed as a Weierstrass representation in $\mathrm{Sol}_{3}$.

Theorem 3. Let $x: \Sigma \rightarrow \mathrm{Sol}_{3}$ be a conformal minimal immersion and $g: \Sigma \rightarrow \overline{\mathbb{C}}$ its Gauss map. Then, whenever $g$ is neither real nor purely imaginary, it is nowhere antiholomorphic $\left(g_{z} \neq 0\right.$ for every point for any local conformal parameter $z$ on $\left.\Sigma\right)$, and it satisfies the second order elliptic equation

$$
g_{z \bar{z}}=\frac{2 g g_{z} g_{\bar{z}}}{g^{2}-\bar{g}^{2}} .
$$

Moreover, the immersion $x=\left(x_{1}, x_{2}, x_{3}\right)$ can be expressed in terms of $g$ by the representation formulas

$$
x_{1 z}=e^{-x_{3}} \frac{\left(\bar{g}^{2}-1\right) g_{z}}{g^{2}-\bar{g}^{2}}, \quad x_{2 z}=i e^{x_{3}} \frac{\left(\bar{g}^{2}+1\right) g_{z}}{g^{2}-\bar{g}^{2}}, \quad x_{3 z}=\frac{2 \bar{g} g_{z}}{g^{2}-\bar{g}^{2}}
$$

whenever it is well-defined.

Conversely, given a map $g: \Sigma \rightarrow \overline{\mathbb{C}}$ defined on a simply connected Riemann surface $\Sigma$ satisfying (6), then the map $x: \Sigma \rightarrow \mathrm{Sol}_{3}$ given by the representation formulas (7) is a conformal minimal immersion with possibly branched points whenever it is well-defined, and its Gauss map is $g$. 
Remark. (1) There exists a similar result for the case of CMC $H$-surfaces ; see [Daniel and Mira 2013].

(2) Equation (6) is the harmonic map equation for maps $g: \Sigma \rightarrow\left(\overline{\mathbb{C}}, d s^{2}\right)$ equipped with the metric

$$
d s^{2}=\frac{|d \omega|^{2}}{\left|\omega^{2}-\bar{\omega}^{2}\right|} .
$$

This is a singular metric, not defined on the real and pure imaginary axes. See [Inoguchi and Lee 2008] for more details.

(3) Equation (6) can be only considered at points where $g \neq \infty$. But if $g$ is a solution of (6), $i / g$ is also a solution at points where $g \neq 0$. The conjugate map $\bar{g}$ and every $g \circ \phi$, with $\phi$ a locally injective holomorphic function, are solutions too. Moreover, if $g$ is a nowhere antiholomorphic solution of (6), and $x$ is the induced conformal minimal immersion, then $i g$ and $1 / g$ induce conformal minimal immersions given by $\sigma x$ and $\tau x$. Finally, $\bar{g}$ is the Gauss map of $\sigma^{2} \tau x$ after a change of orientation.

Definition. The Hopf differential of the map $g$ is the quadratic form

$$
Q=q d z^{2}=\frac{g_{z} \bar{g}_{z}}{g^{2}-\bar{g}^{2}} d z^{2} .
$$

Remark. (1) The function $q$ depends on the choice of coordinates, whereas $Q$ does not.

(2) As stated in the introduction, the Hopf differential (or its Abresch-Rosenberg generalization) is not defined on $\mathrm{Sol}_{3}$. If we apply the definition of the Hopf differential of the harmonic maps on $\left(\overline{\mathbb{C}}, d s^{2}\right)$, we get

$$
Q=\frac{g_{z} \bar{g}_{z}}{\left|g^{2}-\bar{g}^{2}\right|} d z^{2}
$$

but this leads to a nonsmooth differential. Because $g^{2}-\bar{g}^{2}$ is purely imaginary on each quarter of the complex plane, the definitions are related by multiplication by $i$ or $-i$, depending on the quarter. Thus, this "Hopf differential" is defined and holomorphic only on each of the four quarters delimited by the real and purely imaginary axes.

\section{Construction of the helicoids in $\mathrm{Sol}_{3}$}

In this section we construct a one-parameter family of helicoids in $\mathrm{Sol}_{3}$ : we define a helicoid to be a minimal surface containing the $x_{3}$-axis whose intersection with every plane $\left\{x_{3}=\right.$ constant $\}$ is a straight line and which is invariant by left multiplication by an element of $\mathrm{Sol}_{3}$ of the form $(0,0, T)$ for some $T \neq 0$. 
Theorem 4. There exists a one-parameter family $\left(\mathcal{H}_{K}\right)_{K \in]-1 ; 1[\backslash\{0\}}$ of properly embedded minimal helicoids in $\mathrm{Sol}_{3}$ having the following properties:

(1) For all $K \in]-1 ; 1\left[\backslash\{0\}\right.$, the surface $\mathcal{H}_{K}$ contains the $x_{3}$-axis.

(2) For all $K \in]-1 ; 1\left[\backslash\{0\}\right.$, the intersection of $\mathcal{H}_{K}$ and any horizontal plane $\left\{x_{3}=\lambda\right\}$ is a straight line.

(3) For all $K \in]-1 ; 1\left[\backslash\{0\}\right.$, there exists $T_{K}$ such that $\mathcal{H}_{K}$ is invariant by left multiplication by $\left(0,0, T_{K}\right)$.

(4) The helicoids $\mathcal{H}_{K}$ have three symmetries fixing the origin: rotation by $\pi$ around the $x_{3}$-axis, rotation by $\pi$ around the $(x, x, 0)$-axis and rotation by $\pi$ around the $(x,-x, 0)$-axis.

Let $K \in]-1,1[$; we define a map $g: \mathbb{C} \rightarrow \overline{\mathbb{C}}$ by

$$
g(z=u+i v)=e^{-u} e^{i b(v)} e^{-i \pi / 4},
$$

where $b$ satisfies the ODE

$$
b^{\prime}=\sqrt{1-K \cos (2 b)}, \quad b(0)=0 .
$$

Proposition 5. The map $b$ is well-defined and has the following properties:

(1) The function $b$ is an increasing diffeomorphism from $\mathbb{R}$ onto $\mathbb{R}$.

(2) The function $b$ is odd.

(3) There exists a real number $W>0$ such that

$$
\forall v \in \mathbb{R}, \quad b(v+W)=b(v)+\pi .
$$

(4) The function $b$ satisfies $b(k W)=k \pi$, for all $k \in \mathbb{Z}$.

Proof. Since $K \in]-1,1[$, there exists $r>0$ such that $1-K \cos (2 b) \in] r, 2[$; the Cauchy-Lipschitz theorem can be applied, and $b$ is well-defined. By (8) we have $b^{\prime}>0$ on its domain of definition, and $\sqrt{r}<b^{\prime}<2$. Since $b^{\prime}$ is bounded by two positive constants, $b$ is defined on $\mathbb{R}$, and

$$
\lim _{v \rightarrow \pm \infty} b(v)= \pm \infty
$$

The function $\hat{b}: v \mapsto-b(-v)$ satisfies (8) with $\hat{b}(0)=0$; hence $\hat{b}=b$ and $b$ is odd. Finally, there exists $W>0$ such that $b(W)=\pi$; then the function $\tilde{b}: v \mapsto b(v+W)-\pi$ satisfies (8) with $\tilde{b}(0)=0$; hence, $\tilde{b}=b$.

Corollary 6. We have $b(k W / 2)=k \pi / 2$ for all $k \in 2 \mathbb{Z}+1$.

Proof. We have

$$
b\left(\frac{W}{2}\right)=b\left(-\frac{W}{2}+W\right)=-b\left(\frac{W}{2}\right)+\pi,
$$

which gives the formula for $k=1$, and the corollary easily follows. 
Proposition 7. The function $g$ satisfies $\left(g^{2}-\bar{g}^{2}\right) g_{z \bar{z}}=2 g g_{z} g_{\bar{z}}$, and its Hopf differential is

$$
Q=\frac{i K}{8} d z^{2}
$$

Proof. A direct calculation shows that $g$ satisfies the equation. Hence, the Hopf differential is given by

$$
Q=\frac{g_{z} \bar{g}_{z}}{g^{2}-\bar{g}^{2}} d z^{2}=\frac{i\left(1-b^{\prime 2}\right)}{8 \cos (2 b)} d z^{2}=\frac{i K}{8} d z^{2} .
$$

Thus the map $g$ induces a conformal minimal immersion $x=\left(x_{1}, x_{2}, x_{3}\right)$ such that

$$
\begin{aligned}
& x_{1 z}=e^{-x_{3}} \frac{\left(\bar{g}^{2}-1\right) g_{z}}{g^{2}-\bar{g}^{2}}=\frac{\left(1+i e^{-2 u} e^{-2 i b}\right)\left(1-b^{\prime}\right) e^{i b} e^{i \pi / 4}}{4 e^{-u} \cos (2 b)} e^{-x_{3}}, \\
& x_{2 z}=i e^{x_{3}} \frac{\left(\bar{g}^{2}+1\right) g_{z}}{g^{2}-\bar{g}^{2}}=-\frac{\left(1-i e^{-2 u} e^{-2 i b}\right) i\left(1-b^{\prime}\right) e^{i b} e^{i \pi / 4}}{4 e^{-u} \cos (2 b)} e^{x_{3}}, \\
& x_{3 z}=\frac{2 \bar{g} g_{z}}{g^{2}-\bar{g}^{2}}=\frac{i\left(b^{\prime}-1\right)}{2 \cos (2 b)} .
\end{aligned}
$$

This map is an immersion since the metric induced by $x$ is given by

$$
d w^{2}=\left\|x_{u}\right\|^{2}|d z|^{2}=\frac{K^{2}}{\left(1+b^{\prime}\right)^{2}} \cosh ^{2} u|d z|^{2} .
$$

We obtain immediately that $x_{3}$ is a one-variable function and satisfies

$$
x_{3}^{\prime}(v)=\frac{1-b^{\prime}(v)}{\cos (2 b(v))}=\frac{K}{1+b^{\prime}(v)} .
$$

Remark. For $K=0$, we get $x_{3}$ is constant, and the image of $x$ is a point. In the sequel, we will always exclude this case.

By setting $x_{3}(0)=0$, we choose $x_{3}$ among the primitive functions.

Proposition 8. (1) The function $x_{3}$ is defined on $\mathbb{R}$ and is bijective.

(2) The function $x_{3}$ is odd.

(3) The function $x_{3}$ satisfies

$$
x_{3}(v+W)=x_{3}(v)+x_{3}(W)
$$

for all real numbers $v$.

Proof. The map $x_{3}$ is bijective on $\mathbb{R}$ since it is a primitive of a continuous function, and its derivative has the sign of $K$. Since the map $b$ is odd, $b^{\prime}$ is even, so $x_{3}^{\prime}$ is even and $x_{3}$ is odd. Finally, we have $x_{3}^{\prime}(v+W)=x_{3}^{\prime}(v)$, and the result follows. 
Hence, the functions

$$
\begin{aligned}
& x_{1}(u+i v)=\frac{\sqrt{2}}{2}(\cos b(v)-\sin b(v)) x_{3}^{\prime} e^{-x_{3}} \sinh u, \\
& x_{2}(u+i v)=\frac{\sqrt{2}}{2}(\cos b(v)+\sin b(v)) x_{3}^{\prime} e^{x_{3}} \sinh u,
\end{aligned}
$$

satisfy the equations above.

Theorem 9. Let $K$ be a real number such that $|K|<1$ and $K \neq 0$, and $b$ the function defined by (8). We define the function $x_{3}$ by

$$
x_{3}^{\prime}=\frac{K}{1+b^{\prime}}, \quad x_{3}(0)=0 .
$$

Then the map

$$
x: u+i v \in \mathbb{C} \longmapsto\left(\begin{array}{c}
\frac{\sqrt{2}}{2}(\cos b(v)-\sin b(v)) x_{3}^{\prime} e^{-x_{3}} \sinh u \\
\frac{\sqrt{2}}{2}(\cos b(v)+\sin b(v)) x_{3}^{\prime} e^{x_{3}} \sinh u \\
x_{3}(v)
\end{array}\right)
$$

is a conformal minimal immersion whose Gauss map is

$$
g: u+i v \in \mathbb{C} \longmapsto e^{-u} e^{i b(v)} e^{-i \pi / 4} .
$$

Moreover,

$$
\left(0,0,2 x_{3}(W)\right) * x(u+i v)=x(u+i(v+2 W))
$$

for all $u, v \in \mathbb{R}$. The surface given by $x$ is called $a$ helicoid of parameter $K$ and will be denoted by $\mathcal{H}_{K}$.

Proof. Equation (10) means that the helicoid is invariant by left multiplication by $\left(0,0,2 x_{3}(W)\right)$. Recall that we have the identity

$$
x_{3}(v+2 W)=x_{3}(v+W)+x_{3}(W)=x_{3}(v)+2 x_{3}(W)
$$

for all real numbers $v$. Thus we get the result for the third coordinate and we prove in the same way that $e^{-2 x_{3}(W)} x_{1}(u+i v)=x_{1}(u+i(v+2 W))$ and $e^{2 x_{3}(W)} x_{2}(u+i v)=x_{2}(u+i(v+2 W))$.

Remark. (1) The surface $\mathcal{H}_{K}$ is embedded because $x_{3}$ is bijective. It is easy to see that it is even properly embedded.

(2) The surfaces $\mathcal{H}_{K}$ and $\mathcal{H}_{-K}$ are related; if we denote by the indices $K$ and $K^{\prime}$ the data describing $\mathcal{H}_{K}$ and $\mathcal{H}_{-K}$, we get

$$
\left\{\begin{array}{c}
b_{-K}(v)=b_{K}(v+W / 2)-\pi / 2, \\
x_{3-K}(v)=-x_{3 K}(v+W / 2)+x_{3 K}(W / 2) .
\end{array}\right.
$$


In particular, $x_{3-K}(W)=-x_{3 K}(W)$ and both surfaces have the same period $\left|x_{3 K}(W)\right|$. Finally,

$$
x_{-K}(u+i v)=\left(0,0, x_{3 K}(W / 2)\right) * \sigma^{3} x_{K}(u+i(v+W / 2)) .
$$

Thus, there exists an isometry of $\mathrm{Sol}_{3}$ that puts $\mathcal{H}_{-K}$ on $\mathcal{H}_{K}$.

Proposition 10. For every real number $T$, there exists a unique helicoid $\mathcal{H}_{K}$ (up to isometry, i.e., up to $K \leftrightarrow-K$ ) whose period is $T$.

Proof. We noticed that the period of the helicoid $\mathcal{H}_{K}$ is

$$
\begin{aligned}
2 x_{3}(W):=2 x_{3 K}(W) & =2 \int_{0}^{W} \frac{K}{1+b^{\prime}(s)} d s \\
& =2 K \int_{0}^{\pi} \frac{d u}{\sqrt{1-K \cos (2 u)}(1+\sqrt{1-K \cos (2 u)})},
\end{aligned}
$$

with the change of variables $u=b(s)$ and $b(W)=\pi$. Seeing $x_{3 K}(W)$ as a function of the variable $K$, we get

$$
\frac{\partial x_{3 K}(W)}{\partial K}=\int_{0}^{\pi} \frac{1}{(1-K \cos (2 u))^{3 / 2}} d u
$$

(valid for $K$ in every compact set $[0, a] \subset[0,1[$, and so in $[0,1[$ ). Then the function $K \mapsto x_{3 K}(W)$ is injective. Moreover, we have $x_{30}(W)=0$ and

$$
\begin{aligned}
x_{31}(W) & =\int_{0}^{\pi} \frac{1}{\sqrt{1-\cos (2 u)}(1+\sqrt{1-\cos (2 u)})} d u \\
& =\int_{0}^{\pi} \frac{1}{\sqrt{2} \sin u(1+\sqrt{2} \sin u)} d u \\
& =\frac{1}{\sqrt{2}} \int_{0}^{\infty} \frac{1+t^{2}}{1+2 \sqrt{2} t+t^{2}} d t=+\infty,
\end{aligned}
$$

so $x_{3 K}(W)$ is a bijection from $] 0,1[$ onto $] 0,+\infty[$.

The vector field defined by

$$
\begin{aligned}
N & =\frac{1}{1+|g|^{2}}\left[\begin{array}{c}
2 \Re(g) \\
2 \Im(g) \\
1-|g|^{2}
\end{array}\right] \\
& =\frac{\sqrt{2}}{2 \cosh u}\left[\begin{array}{c}
\cos b+\sin b \\
\sin b-\cos b \\
\sqrt{2} \sinh u
\end{array}\right]
\end{aligned}
$$


is normal to the surface. We get

$$
\begin{aligned}
& \nabla_{x_{u}} N=-\sin (2 b) \frac{\sinh u}{\cosh u} x_{u}+\left(\frac{1+b^{\prime}}{K \cosh ^{2} u}-\cos (2 b)\right) x_{v}, \\
& \nabla_{x_{v}} N=\left(\frac{1+b^{\prime}}{K \cosh ^{2} u}-\cos (2 b)\right) x_{u}+\sin (2 b) \frac{\sinh u}{\cosh u} x_{v},
\end{aligned}
$$

and thus the Gauss curvature is given by

$$
\mathcal{K}=-1+\frac{1}{\cosh ^{2} u}\left(\frac{2\left(1+b^{\prime}\right) \cos (2 b)}{K}-\frac{\left(1+b^{\prime}\right)^{2}}{K^{2} \cosh ^{2} u}+\sin ^{2}(2 b)\right) .
$$

In particular, the fundamental pieces of the helicoids have infinite total curvature since

$\mathcal{K} d A=\left(-\frac{K^{2}}{\left(1+b^{\prime}\right)^{2}} \cosh ^{2} u+\frac{2 K \cos (2 b)}{1+b^{\prime}}-\frac{1}{\cosh ^{2} u}+\frac{K^{2} \sin ^{2}(2 b)}{\left(1+b^{\prime}\right)^{2}}\right) d u d v$.

We notice that

$$
x(-u+i v)=\left(\begin{array}{c}
-x_{1}(u+i v) \\
-x_{2}(u+i v) \\
x_{3}(v)
\end{array}\right)=\sigma^{2} x(u+i v),
$$

where $\sigma$ and $\tau$ are the isometries introduced in the first section: the helicoid $\mathcal{H}_{K}$ is symmetric by rotation by $\pi$ around the $x_{3}$-axis, which is included in the helicoid as the image by $x$ of the purely imaginary axis of $\mathbb{C}$. On this axis we have

$$
g(0+i v)=-i e^{i b(v)} .
$$

Hence, the straight line $\{(x, x, 0) \mid x \in \mathbb{R}\}$ is included in the helicoid as the image by $x$ of the real line. Along this line, we have

$$
g(u+i 0)=e^{-u} e^{-i \pi / 4} .
$$

Then we notice that

$$
x(u-i v)=\left(\begin{array}{c}
x_{2}(u+i v) \\
x_{1}(u+i v) \\
-x_{3}(v)
\end{array}\right)=\sigma \tau x(u+i v) .
$$

Thus, $\mathcal{H}_{K}$ is symmetric by rotation by $\pi$ around the axis $\{(x, x, 0) \mid x \in \mathbb{R}\}$.

Remark. The straight line $\{(x, x, 0) \mid x \in \mathbb{R}\}$ is a geodesic of the helicoid. It's even a geodesic of $\mathrm{Sol}_{3}$. 


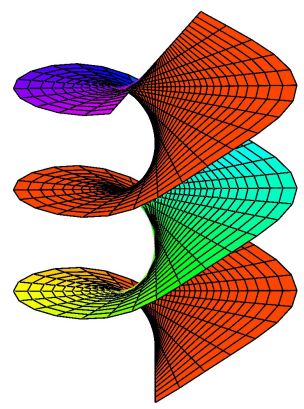

Figure 1. Helicoid for $K=0.4$, created with Scilab.

Since the function sinh is odd, we deduce that

$$
x(-u-i v)=\left(\begin{array}{c}
-x_{2}(u+i v) \\
-x_{1}(u+i v) \\
-x_{3}(v)
\end{array}\right)=\sigma^{3} \tau x(u+i v) .
$$

Thus, $\mathcal{H}_{K}$ is symmetric by rotation by $\pi$ around the axis $\{(x,-x, 0) \mid x \in \mathbb{R}\}$ (but this axis is not included in the surface).

The helicoid $\mathcal{H}_{K}$ has no more symmetry fixing the origin; indeed if it did, there would exist a diffeomorphism $\phi$ of $\mathbb{C}$ such that $x \circ \phi=\sigma^{2} \circ x$ (we choose $\sigma^{2}$ as an example, but it is the same idea for the other elements of the isotropy group of the origin of $\mathrm{Sol}_{3}$ ). By composition, the surface would have every symmetry of the isotropy group. But if $x \circ \phi=\tau x$, the decomposition $\phi=\phi_{1}+i \phi_{2}$ leads to

$$
\left(\begin{array}{c}
x_{1}\left(\phi_{1}(u+i v)+i \phi_{2}(u+i v)\right) \\
x_{2}\left(\phi_{1}(u+i v)+i \phi_{2}(u+i v)\right) \\
x_{3}\left(\phi_{2}(u+i v)\right)
\end{array}\right)=\left(\begin{array}{c}
-x_{1}(u+i v) \\
x_{2}(u+i v) \\
x_{3}(v)
\end{array}\right) .
$$

Because $x_{3}$ is bijective, we get $\phi_{2}(u+i v)=v$ for all $u, v$, and then we get at the same time $\sinh \left(\phi_{1}(u+i v)\right)=\sinh u$ and $\sinh \left(\phi_{1}(u+i v)\right)=-\sinh u$, which is impossible.

\section{Catenoids in $\mathrm{Sol}_{3}$}

In this section we construct examples of minimal annuli in $\mathrm{Sol}_{3}$. Let $\left.\alpha \in\right]-1 ; 1$. We start from a map $g$ defined on $\mathbb{C}$ by

$$
g(z=u+i v)=-i e^{-u-\gamma(v)} e^{i \rho(v)},
$$

where $\rho$ satisfies the ODE

$$
\rho^{\prime}=\sqrt{1-\alpha^{2} \sin ^{2}(2 \rho)}, \quad \rho(0)=0,
$$


and $\gamma$ is defined by

$$
\gamma^{\prime}=-\alpha \sin (2 \rho), \quad \gamma(0)=0 .
$$

Proposition 11. The map $\rho$ is well-defined and has the following properties:

(1) The function $\rho$ is an increasing diffeomorphism from $\mathbb{R}$ onto $\mathbb{R}$.

(2) The function $\rho$ is odd.

(3) There exists a real number $V>0$ such that

$$
\forall v \in \mathbb{R}, \quad \rho(v+V)=\rho(v)+\pi .
$$

(4) The function $\rho$ satisfies $\rho(k V)=k \pi$ for all $k \in \mathbb{Z}$.

Proof. Since $\alpha \in]-1,1\left[\right.$, there exists $r>0$ such that $\left.\left.1-\alpha^{2} \sin ^{2}(2 \rho) \in\right] r, 1\right]$; the Cauchy-Lipschitz theorem can be applied, and $\rho$ is well-defined. By (11) we have $\rho^{\prime}>0$ on its domain of definition, and $\sqrt{r}<\rho^{\prime}<1$. Since $\rho^{\prime}$ is bounded by two positive constants, $\rho$ is defined on $\mathbb{R}$, and

$$
\lim _{v \rightarrow \pm \infty} \rho(v)= \pm \infty
$$

The function $\hat{\rho}: v \mapsto-\rho(-v)$ satisfies (11) with $\hat{\rho}(0)=0$; hence $\hat{\rho}=\rho$ and $\rho$ is odd. Finally, there exists $V>0$ such that $\rho(V)=\pi$; Then the function $\tilde{\rho}: v \mapsto \rho(v+V)-\pi$ satisfies (11) with $\tilde{\rho}(0)=0$; hence $\tilde{\rho}=\rho$.

Corollary 12. (1) We have $\rho(k V / 2)=k \pi / 2$ for all $k \in 2 \mathbb{Z}+1$.

(2) We have $\rho(-v+V / 2)=-\rho(v)+\frac{\pi}{2}$ for all $v \in \mathbb{R}$. In particular, $\rho(V / 4)=\frac{\pi}{4}$ and $\rho(3 V / 4)=\frac{3 \pi}{4}$.

Proof. (1) We have

$$
\rho\left(\frac{V}{2}\right)=\rho\left(-\frac{V}{2}+V\right)=-\rho\left(\frac{V}{2}\right)+\pi,
$$

which gives the formula for $k=1$, and part (1) easily follows.

(2) The functions $\rho^{*}: v \mapsto \pi / 2-\rho(-v+V / 2)$ and $\rho$ satisfy equation (11) with $\rho^{*}(0)=\rho(0)=0$, so $\rho^{*}=\rho$ and

$$
\rho(V / 4)=\rho^{*}(V / 4)=\frac{\pi}{2}-\rho\left(\frac{\pi}{2}-\frac{\pi}{4}\right),
$$

and the result follows.

Proposition 13. The function $g$ satisfies $\left(g^{2}-\bar{g}^{2}\right) g_{z \bar{z}}=2 g g_{z} g_{\bar{z}}$, and its Hopf differential is

$$
Q=-\frac{\alpha}{4} d z^{2}
$$


Proof. A direct calculation shows that $g$ satisfies the equation. Hence, the Hopf differential is given by

$$
\widetilde{Q}=\frac{i\left(1-\rho^{\prime 2}-\gamma^{\prime 2}-2 i \gamma^{\prime}\right)}{8 \sin (2 \rho)} d z^{2}=-\frac{\alpha}{4} d z^{2} .
$$

Thus the map $g$ induces a conformal minimal immersion $x=\left(x_{1}, x_{2}, x_{3}\right)$ such that

$$
x_{1 z}=e^{-x_{3}} \frac{\left(\bar{g}^{2}-1\right) g_{z}}{g^{2}-\bar{g}^{2}}, \quad x_{2 z}=i e^{x_{3}} \frac{\left(\bar{g}^{2}+1\right) g_{z}}{g^{2}-\bar{g}^{2}}, \quad x_{3 z}=\frac{2 \bar{g} g_{z}}{g^{2}-\bar{g}^{2}} .
$$

This application is an immersion since the metric induced by $x$ is given by

$$
\begin{aligned}
d w^{2} & =\left\|x_{u}\right\|^{2}|d z|^{2} \\
& =\left(F^{\prime 2}+\alpha^{2}\right) \cosh ^{2}(u+\gamma)|d z|^{2} \\
& =\left(\frac{\alpha^{4} \sin ^{2}(2 \rho)}{\left(1+\rho^{\prime}\right)^{2}}+\alpha^{2}\right) \cosh ^{2}(u+\gamma)|d z|^{2} \\
& =\frac{2 \alpha^{2}}{1+\rho^{\prime}} \cosh ^{2}(u+\gamma)|d z|^{2} .
\end{aligned}
$$

In particular,

$$
x_{3 z}=\frac{i \rho^{\prime}-\gamma^{\prime}-i}{2 \sin (2 \rho)}
$$

that is,

$$
\left\{\begin{array}{l}
x_{3 u}=2 \Re\left(x_{3 z}\right)=-\frac{\gamma^{\prime}}{\sin (2 \rho)}=\alpha, \\
x_{3 v}=-2 \Im\left(x_{3 z}\right)=\frac{1-\rho^{\prime}}{\sin (2 \rho)}=\frac{\alpha^{2} \sin (2 \rho)}{1+\rho^{\prime}} .
\end{array}\right.
$$

Thus

$$
x_{3}(u+i v)=\alpha u+\alpha^{2} \int^{v} \frac{\sin (2 \rho(s))}{1+\rho^{\prime}(s)} d s .
$$

Here we have to choose an initial condition; we set

$$
F(v)=\alpha^{2} \int_{0}^{v} \frac{\sin (2 \rho(s))}{1+\rho^{\prime}(s)} d s,
$$

and define

$$
x_{3}(u+i v)=\alpha u+F(v) .
$$

The function $F$ is well-defined on $\mathbb{R}$.

Proposition 14. The function $F$ is even and $V$-periodic. 
Proof. The function $F^{\prime}$ is odd because $\rho$ is odd and $\rho^{\prime}$ is even, so $F$ is even. Then we get

$$
F^{\prime}(v+V)=\alpha^{2} \frac{\sin (2 \rho(v)+2 \pi)}{1+\rho^{\prime}(v)}=F^{\prime}(v),
$$

so there exists a constant $C$ such that $F(v+V)=F(v)+C$ for all $v \in \mathbb{R}$. By evaluating at zero, we get $C=F(V)$, that is,

$$
\begin{aligned}
C & =\alpha^{2} \int_{0}^{V} \frac{\sin (2 \rho(s))}{1+\rho^{\prime}(s)} d s=\alpha^{2} \int_{0}^{V} H(s) d s \\
& =\alpha^{2}\left\{\int_{0}^{V / 4} H(s) d s+\int_{V / 4}^{3 V / 4} H(s) d s+\int_{3 V / 4}^{V} H(s) d s\right\} \\
& :=\sum_{k=0}^{2} L_{k}(\alpha) .
\end{aligned}
$$

We can now do the change of variable $u=\sin (2 \rho(s))$ in each integral $L_{k}(\alpha)$, with

$$
d u=2 \rho^{\prime}(s) \cos (2 \rho(s)) d s=2(-1)^{k} \sqrt{\left(1-\alpha^{2} u^{2}\right)\left(1-u^{2}\right)} d s .
$$

Thus,

$$
C=\alpha^{2} \int_{-1}^{1} \frac{u d u}{\left(1+\sqrt{1-\alpha^{2} u^{2}}\right) \sqrt{\left(1-\alpha^{2} u^{2}\right)\left(1-u^{2}\right)}}=0
$$

and $F$ is $V$-periodic.

Proposition 15. The function $\gamma$ is even and $V$-periodic.

Proof. We prove the proposition in exactly the same way as for the function $F$.

The two other equations become

$$
\begin{aligned}
& x_{1 z}=e^{-x_{3}} \frac{\left(e^{-u-\gamma-i \rho}+e^{u+\gamma+i \rho}\right)\left(1-\rho^{\prime}-i \gamma^{\prime}\right)}{4 \sin (2 \rho)}, \\
& x_{2 z}=-e^{x_{3}} \frac{\left(e^{u+\gamma+i \rho}-e^{-u-\gamma-i \rho}\right)\left(i-i \rho^{\prime}+\gamma^{\prime}\right)}{4 \sin (2 \rho)} .
\end{aligned}
$$

Those equations lead to

$$
\begin{aligned}
& x_{1}=e^{-\alpha u-F}\left(\frac{e^{u+\gamma}}{2(1-\alpha)}\left(F^{\prime} \cos \rho-\alpha \sin \rho\right)-\frac{e^{-u-\gamma}}{2(1+\alpha)}\left(\alpha \sin \rho+F^{\prime} \cos \rho\right)\right), \\
& x_{2}=e^{\alpha u+F}\left(\frac{-e^{u+\gamma}}{2(1+\alpha)}\left(\alpha \cos \rho+F^{\prime} \sin \rho\right)+\frac{e^{-u-\gamma}}{2(\alpha-1)}\left(\alpha \cos \rho-F^{\prime} \sin \rho\right)\right) .
\end{aligned}
$$

Remark. If $\alpha=0$, then $x(\mathbb{C})=\{0\}$. This case will be excluded in the sequel. 
Theorem 16. Let $\alpha$ be a real number such that $|\alpha|<1$ and $\alpha \neq 0$, and $\rho$ and $\gamma$ the functions defined by (11) and (12). We define the function $F$ by

$$
F(v)=\alpha^{2} \int_{0}^{v} \frac{\sin (2 \rho(s))}{1+\rho^{\prime}(s)} d s .
$$

Then the map $x: \mathbb{C} \rightarrow \mathrm{Sol}_{3}$ defined by

$$
\left(\begin{array}{c}
e^{-\alpha u-F}\left(\frac{e^{u+\gamma}}{2(1-\alpha)}\left(F^{\prime} \cos \rho-\alpha \sin \rho\right)-\frac{e^{-u-\gamma}}{2(1+\alpha)}\left(\alpha \sin \rho+F^{\prime} \cos \rho\right)\right) \\
e^{\alpha u+F}\left(\frac{-e^{u+\gamma}}{2(1+\alpha)}\left(\alpha \cos \rho+F^{\prime} \sin \rho\right)+\frac{e^{-u-\gamma}}{2(\alpha-1)}\left(\alpha \cos \rho-F^{\prime} \sin \rho\right)\right) \\
\alpha u+F
\end{array}\right)
$$

is a conformal minimal immersion whose Gauss map is

$$
g: u+i v \in \mathbb{C} \longmapsto-i e^{-u-\gamma(v)} e^{i \rho(v)} .
$$

Moreover,

$$
x(u+i(v+2 V))=x(u+i v)
$$

for all $u, v \in \mathbb{R}$. The surface given by $x$ is called $a$ catenoid of parameter $\alpha$ and will be denoted by $\mathcal{C}_{\alpha}$.

Proof. The periodicity of $\mathcal{C}_{\alpha}$ is an application of Propositions 11, 14 and 15.

Remark. The surfaces $\mathcal{C}_{\alpha}$ and $\mathcal{C}_{-\alpha}$ are related; if we denote by the indices $\alpha$ and $-\alpha$ the data describing $\mathcal{C}_{\alpha}$ and $\mathcal{C}_{-\alpha}$, we get

$$
\left\{\begin{array}{l}
\rho_{-\alpha}=\rho_{\alpha}, \\
F_{-\alpha}=F_{\alpha}, \\
\gamma_{-\alpha}=-\gamma_{\alpha} .
\end{array}\right.
$$

Thus, we get

$$
x_{-\alpha}(-u+i v)=\sigma^{2} x_{\alpha}(u+i v) .
$$

In particular, there exists an orientation-preserving isometry of $\mathrm{Sol}_{3}$ fixing the origin that sends $\mathcal{C}_{\alpha}$ on $\mathcal{C}_{-\alpha}$.

Now we show that the catenoids are embedded:

Proposition 17. For all $\lambda \in \mathbb{R}$, the intersection of $\mathcal{C}_{\alpha}$ with the plane $\left\{x_{3}=\lambda\right\}$ is a nonempty closed embedded convex curve.

Proof. This intersection is nonempty: $x(\lambda / \alpha+i 0) \in \mathcal{C}_{\alpha} \cap\left\{x_{3}=\lambda\right\}$. We look at the curve in $\mathbb{C}$ defined by $x_{3}(u+i v)=\alpha u+F(v)=\lambda$, i.e., the curve

$$
c: t \in \mathbb{R} \longmapsto\left(\frac{\lambda-F(t)}{\alpha}, t\right) .
$$


Its image by $x$ is

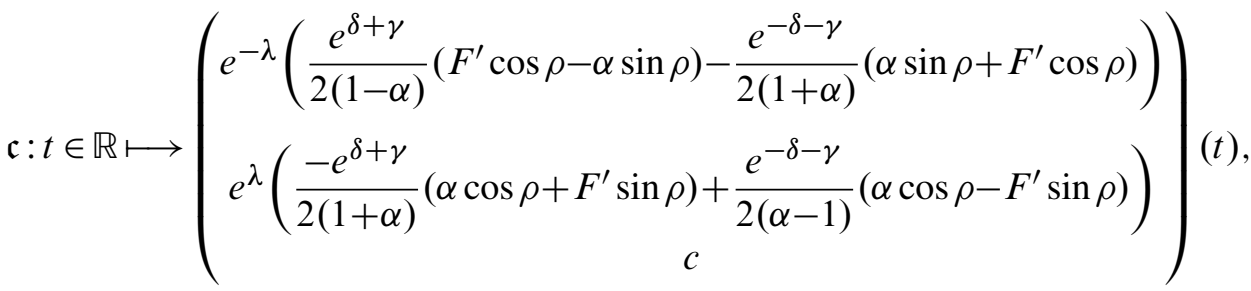

where $\delta=\frac{\lambda-F}{\alpha}$. The calculation leads to

$$
\mathfrak{c}_{1}^{\prime}(t)=\frac{e^{-\lambda}}{\alpha\left(1-\alpha^{2}\right)}\left(A(t) \cosh \left(\frac{\lambda-F}{\alpha}+\gamma\right)+B(t) \sinh \left(\frac{\lambda-F}{\alpha}+\gamma\right)\right),
$$

with

$$
\left\{\begin{array}{r}
A=-F^{2} \cos \rho+\alpha \gamma^{\prime} F^{\prime} \cos \rho-\alpha^{2} \rho^{\prime} \cos \rho+\alpha^{2} F^{\prime} \sin \rho-\alpha^{3} \gamma^{\prime} \sin \rho \\
+\alpha^{2} F^{\prime \prime} \cos \rho-\alpha^{2} F^{\prime} \rho^{\prime} \sin \rho \\
B=\alpha F^{\prime} \sin \rho-\alpha^{2} \gamma^{\prime} \sin \rho+\alpha F^{\prime \prime} \cos \rho-\alpha F^{\prime} \rho^{\prime} \sin \rho-\alpha F^{2} \cos \rho \\
+\alpha^{2} \gamma^{\prime} F^{\prime} \cos \rho-\alpha^{3} \rho^{\prime} \cos \rho .
\end{array}\right.
$$

We remark that $B \equiv 0$ after simplifications, and

$$
A(t)=\left(F^{\prime 2}(t)+\alpha^{2}\right)\left(\alpha^{2}-1\right) \cos \rho(t) .
$$

Finally,

$$
\mathfrak{c}_{1}^{\prime}(t)=-\frac{e^{-\lambda}}{\alpha}\left(F^{\prime 2}(t)+\alpha^{2}\right) \cos \rho(t) \cosh \left(\frac{\lambda-F(t)}{\alpha}+\gamma(t)\right) .
$$

In the same way, we get

$$
\mathfrak{c}_{2}^{\prime}(t)=-\frac{e^{-\lambda}}{\alpha}\left(F^{\prime 2}(t)+\alpha^{2}\right) \sin \rho(t) \cosh \left(\frac{\lambda-F(t)}{\alpha}+\gamma(t)\right) .
$$

Thus

$$
\mathfrak{c}_{1}^{\prime 2}+\mathfrak{c}_{2}^{\prime 2}=\frac{e^{-2 \lambda}}{\alpha^{2}}\left(F^{\prime 2}(t)+\alpha^{2}\right)^{2} \cosh ^{2}\left(\frac{\lambda-F(t)}{\alpha}+\gamma(t)\right)>0,
$$

so the intersection $\mathcal{C}_{\alpha} \cap\left\{x_{3}=\lambda\right\}$ is a smooth curve; moreover, it's closed since $\mathfrak{c}(t+2 V)=\mathfrak{c}(t)$ for all $t \in \mathbb{R}$.

The planes $\left\{x_{3}=\lambda\right\}$ are flat: indeed, the metrics on these planes are $e^{2 \lambda} d x_{1}^{2}+$ $e^{-2 \lambda} d x_{2}^{2}$, so up to an affine transformation, we can work in euclidean coordinates, as we suppose in this proof since affinities preserve convexity.

To prove that $\mathfrak{c}$ is embedded and convex, we consider the part of $\mathfrak{c}$ corresponding to $t \in(-V / 2, V / 2)$. On $(-V / 2, V / 2)$, we have $\cos \rho(t)>0$, thanks to Proposition 11 

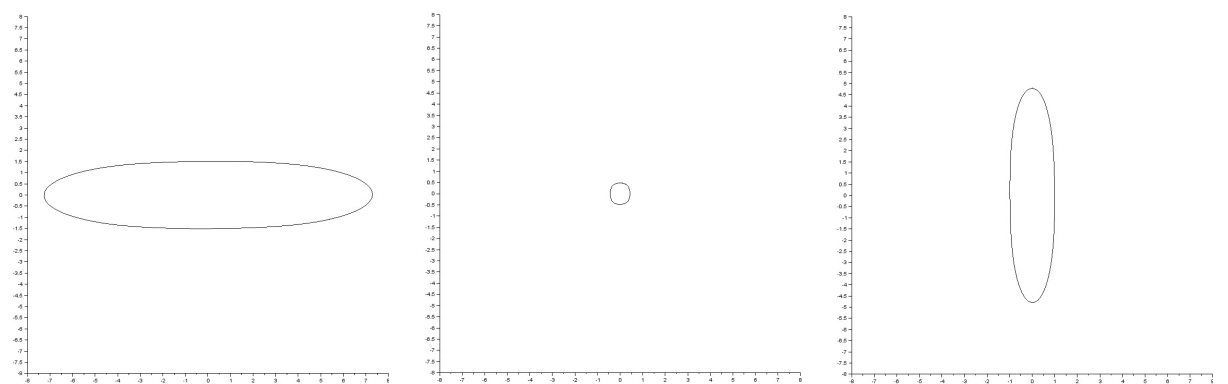

Figure 2. Sections with $\left\{x_{3}=-1\right\},\left\{x_{3}=0\right\}$, and $\left\{x_{3}=1\right\}$, created with Scilab.

and Corollary 12. So $\mathfrak{c}_{1}^{\prime}(t)<0$ if $\alpha>0$ (and $\mathfrak{c}_{1}^{\prime}(t)>0$ if $\alpha<0$ ) and $\mathfrak{c}_{1}$ is injective and decreasing if $\alpha>0$ (and increasing if $\alpha<0$ ). We get

$$
\frac{d \mathfrak{c}_{2}}{d \mathfrak{c}_{1}}=\tan \rho(t)
$$

so $d \mathfrak{c}_{2} / d \mathfrak{c}_{1}$ is an increasing function of $t$, and also of $\mathfrak{c}_{1}$ if $\alpha<0$ (and a decreasing function of the decreasing function $\mathfrak{c}_{1}$ if $\alpha>0$ ). In both cases, the curve is convex.

Then, the half of $\mathfrak{c}$ corresponding to $t \in(-V / 2, V / 2)$ is convex and embedded. Since $\mathfrak{c}(t+V)=-\mathfrak{c}(t)$, the entire curve is convex and embedded.

Figure 2 shows sections of the catenoid $\alpha=-0.6$ with planes $\left\{x_{3}=\right.$ constant $\}$.

Corollary 18. The surface $\mathcal{C}_{\alpha}$ is properly embedded for all $\left.\alpha \in\right]-1,1[\backslash\{0\}$.

Proposition 19. For all $\alpha \in]-1,1\left[\backslash\{0\}\right.$, the surface $\mathcal{C}_{\alpha}$ is conformally equivalent to $\mathbb{C} \backslash\{0\}$.

Proof. The map $x: \mathbb{C} /(2 i V \mathbb{Z}) \rightarrow \mathcal{C}_{\alpha}$ is a conformal bijective parametrization of $\mathcal{C}_{\alpha}$.

The vector field defined by

$$
N=\frac{1}{\cosh u}\left[\begin{array}{c}
e^{-\gamma} \sin \rho \\
-e^{-\gamma} \cos \rho \\
\sinh u
\end{array}\right]
$$

is normal to the surface.

We have

$$
x(u+i(v+V))=\left(\begin{array}{r}
-x_{1}(u+i v) \\
-x_{2}(u+i v) \\
x_{3}(u+i v)
\end{array}\right)=\sigma^{2} x(u+i v) .
$$

Thus, the surface $\mathcal{C}_{\alpha}$ is symmetric by rotation by $\pi$ around the $x_{3}$-axis. 


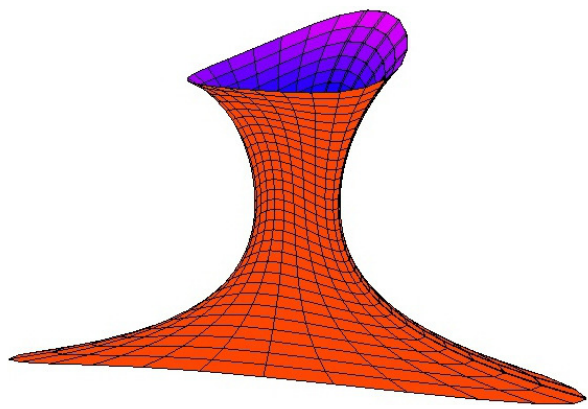

Figure 3. Catenoid for $\alpha=-0.6$, created with Scilab.

Remark. The $x_{3}$-axis is contained in the "interior" of $\mathcal{C}_{\alpha}$ since each curve $\mathcal{C}_{\alpha} \cap\left\{x_{3}=\lambda\right\}$ is convex and symmetric with respect to this axis.

We also get

$$
x(u-i v)=\left(\begin{array}{r}
-x_{1}(u+i v) \\
x_{2}(u+i v) \\
x_{3}(u+i v)
\end{array}\right)=\tau x(u+i v),
$$

and the surface $\mathcal{C}_{\alpha}$ is symmetric by reflection in the plane $\left\{x_{1}=0\right\}$, and finally we have

$$
x(u+i(-v+V))=\sigma^{2} \tau x(u+i v),
$$

and $\mathcal{C}_{\alpha}$ is symmetric by reflection in the plane $\left\{x_{2}=0\right\}$.

If $\mathcal{C}_{\alpha}$ had another symmetry fixing the origin, it would have every symmetry of the isotropy group of $\mathrm{Sol}_{3}$, and we prove as for the helicoid that it is impossible.

\section{Limits of catenoids}

6.1. The case $\alpha=0$. In this part we consider the limit surface of the catenoids $\mathcal{C}_{\alpha}$ when $\alpha$ goes to zero. For this, we do the change of parameters

$$
\left\{\begin{array}{l}
u^{\prime}=u+\ln \alpha, \\
v^{\prime}=v .
\end{array}\right.
$$

In these coordinates, the immersion $x$ given in Theorem 16 takes the form

$$
\left(\begin{array}{c}
e^{\alpha \ln \alpha-\alpha u^{\prime}-F}\left(\frac{e^{u^{\prime}+\gamma}}{2 \alpha(1-\alpha)}\left(\cos \rho F^{\prime}-\alpha \sin \rho\right)-\frac{\alpha e^{-u^{\prime}-\gamma}}{2(1+\alpha)}\left(\alpha \sin \rho+\cos \rho F^{\prime}\right)\right) \\
e^{-\alpha \ln \alpha+\alpha u^{\prime}+F}\left(\frac{-e^{u^{\prime}+\gamma}}{2 \alpha(1+\alpha)}\left(\alpha \cos \rho+F^{\prime} \sin \rho\right)+\frac{\alpha e^{-u^{\prime}-\gamma}}{2(\alpha-1)}\left(\alpha \cos \rho-F^{\prime} \sin \rho\right)\right) \\
-\alpha \ln \alpha+\alpha u^{\prime}+F
\end{array}\right) .
$$


Letting $\alpha$ go to zero, we get

$$
\left\{\begin{aligned}
& \rho \longrightarrow \mathrm{Id}, \\
& F / \alpha \longrightarrow 0 \\
& F^{\prime} / \alpha \longrightarrow 0 \\
& \gamma \longrightarrow 0
\end{aligned}\right.
$$

and so the limit immersion is

$$
\left(\begin{array}{c}
-\frac{e^{u^{\prime}}}{2} \sin v^{\prime} \\
-\frac{e^{u^{\prime}}}{2} \cos v^{\prime} \\
0
\end{array}\right)
$$

Thus, we obtain a parametrization of the plane $\left\{x_{3}=0\right\}$, which is the limit of the family $\left(\mathcal{C}_{\alpha}\right)$ when $\alpha \rightarrow 0$.

6.2. The case $\alpha=1$. We end by the study of the case $\alpha=1$ (the case $\alpha=-1$ is exactly the same). We show that the limit surface is a minimal entire graph:

Proposition 20. Let $\boldsymbol{x}: \mathbb{R}^{2} \rightarrow \mathrm{Sol}_{3}$ be defined by

$$
\boldsymbol{x}(u+i v)=\left(\begin{array}{c}
\boldsymbol{x}_{1} \\
\boldsymbol{x}_{2} \\
\boldsymbol{x}_{3}
\end{array}\right)=\left(\begin{array}{c}
-\frac{\tanh v}{2}\left(1+e^{-2 u}\right) \\
\frac{e^{2 u}}{4}-\frac{u}{2}-\frac{\cosh (2 v)}{4} \\
u+\ln (\cosh v)
\end{array}\right) .
$$

Then $\boldsymbol{x}$ is a minimal immersion and there exists a $C^{\infty}$-function $f$ defined on $\mathbb{R}^{2}$ such that the image of $x$ (called $\mathcal{S}$ ) is the $\boldsymbol{x}_{2}$-graph of $f$ given by $\boldsymbol{x}_{2}=f\left(\boldsymbol{x}_{1}, \boldsymbol{x}_{3}\right)$. Proof. We show that this surface is (up to a translation) the limit surface of the

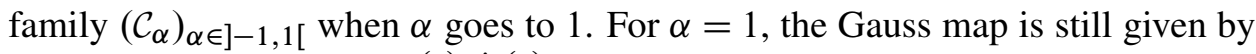
$g(z=u+i v)=-i e^{-u-\gamma(v)} e^{i \rho(v)}$, but $\rho$ satisfies the ODE

$$
\rho^{\prime}=\cos (2 \rho), \quad \rho(0)=0,
$$

and $\gamma$ is still defined by

$$
\gamma^{\prime}=-\sin (2 \rho), \quad \gamma(0)=0 .
$$

We have explicit expressions for these functions, which are given by

$$
\begin{aligned}
& \rho(v)=\arctan e^{2 v}-\pi / 4=\arctan (\tanh v), \\
& \gamma(v)=-\frac{1}{2} \ln (\cosh (2 v)) .
\end{aligned}
$$



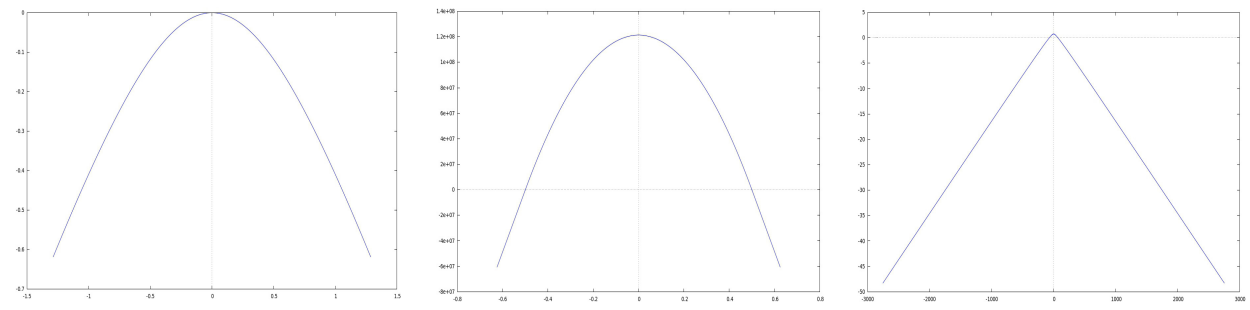

Figure 4. Sections with $\left\{x_{3}=0\right\},\left\{x_{3}=10\right\}$, and $\left\{x_{3}=-2\right\}$, created with Maxima.

Thus by setting

$$
F(v)=\int_{0}^{v} \frac{\sin (2 \rho(s))}{1+\cos (2 \rho(s))} d s,
$$

we obtain $F(v)=\ln (\cosh v)$. Then, the immersion $x$ is given by

$$
x=\left(\begin{array}{c}
-\frac{e^{-2 u}}{2} \tanh v+\frac{e^{-v}}{2 \cosh v} \\
\frac{e^{2 u}}{4}-\frac{u}{2}-\frac{\cosh (2 v)}{4} \\
u+\ln (\cosh v)
\end{array}\right) .
$$

A unit normal vector field is given by

$$
N=\frac{1}{1+e^{-2 u} \cosh (2 v)}\left[\begin{array}{c}
2 e^{-u} \sinh v \\
-2 e^{-u} \cosh v \\
1-e^{-2 u} \cosh (2 v)
\end{array}\right] .
$$

Thus, we get

$$
g(u+i v)=-i e^{-u}(\cosh v+i \sinh v),
$$

which satisfies the harmonic equation (6). The metric induced by this immersion on the surface is

$$
d s^{2}=\left(e^{-4 u} \tanh ^{2} v+e^{2 u} \sinh ^{2} u+1\right)|d z|^{2} .
$$

This surface is symmetric by reflection in the plane $\left\{x_{1}=1 / 2\right\}$ since

$$
x(u+i v)=\left(\begin{array}{c}
\frac{1}{2}-\frac{\tanh v}{2}\left(1+e^{-2 u}\right) \\
\frac{e^{2 u}}{4}-\frac{u}{2}-\frac{\cosh (2 v)}{4} \\
u+\ln (\cosh v)
\end{array}\right)=\left(\begin{array}{c}
\frac{1}{2}+\tilde{x}_{1}(u, v) \\
x_{2}(u, v) \\
x_{3}(u, v)
\end{array}\right),
$$




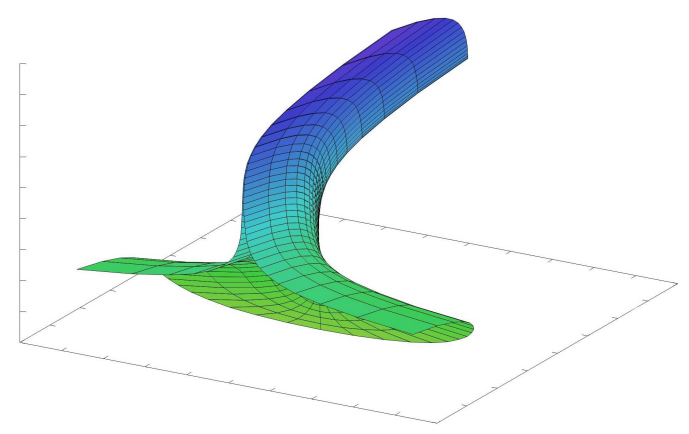

Figure 5. The surface $\mathcal{S}$, created with Maxima.

and so

$$
x(u-i v)=\left(\begin{array}{c}
\frac{1}{2}-\tilde{x}_{1}(u, v) \\
x_{2}(u, v) \\
x_{3}(u, v)
\end{array}\right) .
$$

This property is equivalent to the property that the translated surface $(-1 / 2,0,0) *$ $x(u+i v)$ is symmetric with respect to $\left\{x_{1}=0\right\}$. This translated surface is the image of the immersion $\boldsymbol{x}$ defined by

$$
\boldsymbol{x}(u+i v)=(-1 / 2,0,0) * x(u+i v)=\left(\begin{array}{c}
-\frac{\tanh v}{2}\left(1+e^{-2 u}\right) \\
\frac{e^{2 u}}{4}-\frac{u}{2}-\frac{\cosh (2 v)}{4} \\
u+\ln (\cosh v)
\end{array}\right) .
$$

Then, this surface is analytic (like any minimal surface in $\mathrm{Sol}_{3}$ ), so it is a local analytic $\boldsymbol{x}_{2}$-graph around every point where $\partial_{2}$ doesn't belong to the tangent plane, i.e., $\left\langle N, \partial_{2}\right\rangle \neq 0$. But

$$
\left\langle N, \partial_{2}\right\rangle=0 \Longleftrightarrow \cosh v e^{-u}=0,
$$

which is impossible. Thus, $\mathcal{S}$ is a local analytic $\boldsymbol{x}_{2}$-graph around every point. Then, we consider sections of the surface $\mathcal{S}$ with planes $\left\{\boldsymbol{x}_{3}=\right.$ constant $\}$ : on the plane $\left\{\boldsymbol{x}_{3}=\lambda\right\}$, we get the curve

$$
c_{\lambda}(t)=\left(\begin{array}{c}
-\frac{\tanh t}{2}\left(1+e^{-2 \lambda} \cosh ^{2} t\right) \\
\frac{e^{2 \lambda}}{4 \cosh ^{2} t}-\frac{\lambda}{2}+\frac{\ln (\cosh t)}{2}-\frac{\cosh (2 t)}{4}
\end{array}\right):=\left(\begin{array}{l}
\boldsymbol{x}_{1 \lambda}(t) \\
\boldsymbol{x}_{2 \lambda}(t)
\end{array}\right) .
$$

Then,

$$
\boldsymbol{x}_{1}^{\prime}(t)=\frac{\tanh ^{2} t-1}{2}-\frac{e^{-2 \lambda}}{2}\left(\cosh ^{2} t+\sinh ^{2} t\right)<0
$$


for all $t \in \mathbb{R}$. Thus, the curves are injective, so the surface $\mathcal{S}$ is embedded. Moreover, by the implicit function theorem, we deduce that for every $\lambda \in \mathbb{R}$, there exists a function $f_{\lambda}$ such that $\boldsymbol{x}_{2 \lambda}=f_{\lambda}\left(\boldsymbol{x}_{1 \lambda}\right)$. Because the function $\boldsymbol{x}_{1 \lambda}$ is a decreasing diffeomorphism of $\mathbb{R}$, the function $f_{\lambda}$ is defined on $\mathbb{R}$. This calculus is valid for all $\lambda \in \mathbb{R}$, so there exists a function $f: \mathbb{R}^{2} \rightarrow \mathbb{R}$ such that $\boldsymbol{x}_{2}=f\left(\boldsymbol{x}_{1}, \boldsymbol{x}_{3}\right)$.

Finally, this function $f$ coincides around every point with the local $C^{\infty}$-functions which give the local graphs, and so $f$ is $C^{\infty}$.

As a conclusion, we can notice that, for a fixed $\boldsymbol{x}_{3}$,

- when $x_{1} \rightarrow+\infty, x_{2} \approx-x_{1} e^{2 x_{3}}$;

- when $x_{1} \rightarrow-\infty, x_{2} \approx x_{1} e^{2 x_{3}}$.

\section{References}

[Abresch and Rosenberg 2004] U. Abresch and H. Rosenberg, "A Hopf differential for constant mean curvature surfaces in $\mathbf{S}^{2} \times \mathbb{R}$ and $\mathbf{H}^{2} \times \mathbb{R}$ ”, Acta Math. 193:2 (2004), 141-174. MR 2006h:53003 Zbl 1078.53053

[Abresch and Rosenberg 2005] U. Abresch and H. Rosenberg, "Generalized Hopf differentials", Mat. Contemp. 28 (2005), 1-28. MR 2006h:53004 Zbl 1118.53036

[Berdinskii and Taimanov 2005] D. A. Berdinskii and I. A. Taimanov, "Поверхности в трехмерных пруппах Ли", Sibirsk. Mat. Zh. 46:6 (2005), 1248-1264. Translated as "Surfaces in threedimensional Lie groups" in Sib. Math. J. 46:6 (2005), 1005-1019. MR 2006j:53087 Zbl 1117.53045

[Daniel and Hauswirth 2009] B. Daniel and L. Hauswirth, "Half-space theorem, embedded minimal annuli and minimal graphs in the Heisenberg group", Proc. Lond. Math. Soc. (3) 98:2 (2009), 445-470. MR 2009m:53018 Zbl 1163.53036

[Daniel and Mira 2013] B. Daniel and P. Mira, "Existence and uniqueness of constant mean curvature spheres in Sol 3 ”, J. Reine Angew. Math. 685 (2013), 1-32. MR 3181562 Zbl 06245199

[Inoguchi and Lee 2008] J.-I. Inoguchi and S. Lee, "A Weierstrass type representation for minimal surfaces in Sol”, Proc. Amer. Math. Soc. 136:6 (2008), 2209-2216. MR 2008k:53016 Zbl 1157.53007

[López 2014] R. López, "Invariant surfaces in $\mathrm{Sol}_{3}$ with constant mean curvature and their computer graphics”, Adv. Geom. 14:1 (2014), 31-48. MR 3159090 Zbl 1283.53058

[López and Munteanu 2011] R. López and M. I. Munteanu, "Surfaces with constant mean curvature in Sol geometry”, Differential Geom. Appl. 29:S1 (2011), S238-S245. MR 2012k:53016 Zbl 1250.53058

[López and Munteanu 2012] R. López and M. I. Munteanu, "Minimal translation surfaces in $\mathrm{Sol}_{3}$ ”, J. Math. Soc. Japan 64:3 (2012), 985-1003. MR 2965436 Zbl 1254.53033

[López and Munteanu 2014] R. López and M. I. Munteanu, "Invariant surfaces in the homogeneous space Sol with constant curvature", Math. Nachr. 287:8-9 (2014), 1013-1024. MR 3219227 Zbl 1293.53076

[Masaltsev 2006] L. A. Masaltsev, "Minimal surfaces in standard three-dimensional geometry Sol 3 ", Zh. Mat. Fiz. Anal. Geom. 2:1 (2006), 104-110. MR 2006j:53008 Zbl 1144.53016

[Meeks and Pérez 2012] W. H. Meeks, III and J. Pérez, "Constant mean curvature surfaces in metric Lie groups", pp. 25-110 in Geometric analysis: partial differential equations and surfaces (Granada, 2010), edited by J. Pérez and J. A. Gálvez, Contemporary Mathematics 570, American Mathematical Society, Providence, RI, 2012. MR 2963596 Zbl 1267.53006 
[Menezes 2014] A. Menezes, "Periodic minimal surfaces in semidirect products", J. Aust. Math. Soc. 96:1 (2014), 127-144. MR 3177813 Zbl 1288.53052

[Nguyen 2014] M. H. Nguyen, "The Dirichlet problem for the minimal surface equation in $\mathrm{Sol}_{3}$ with possible inifinite boundary data”, preprint, 2014. arXiv 1312.6194

Received January 22, 2014. Revised August 1, 2014.

Christophe Desmonts

INSTITUT ELIE CARTAN DE LORRAINE

UNIVERSITÉ DE LORRAINE

B.P. 70239

54506 VANDOEUVRE-LÈS-NANCY CEDEX

FRANCE

christophe.desmonts@univ-lorraine.fr 


\title{
PACIFIC JOURNAL OF MATHEMATICS
}

\author{
msp.org/pjm
}

Founded in 1951 by E. F. Beckenbach (1906-1982) and F. Wolf (1904-1989)

\section{EDITORS}

Don Blasius (Managing Editor)

Department of Mathematics

University of California

Los Angeles, CA 90095-1555

blasius@math.ucla.edu

\author{
Paul Balmer \\ Department of Mathematics \\ University of California \\ Los Angeles, CA 90095-1555 \\ balmer@math.ucla.edu \\ Robert Finn \\ Department of Mathematics \\ Stanford University \\ Stanford, CA 94305-2125 \\ finn@math.stanford.edu \\ Sorin Popa \\ Department of Mathematics \\ University of California \\ Los Angeles, CA 90095-1555 \\ popa@math.ucla.edu
}

\author{
Vyjayanthi Chari \\ Department of Mathematics \\ University of California \\ Riverside, CA 92521-0135 \\ chari@math.ucr.edu \\ Kefeng Liu \\ Department of Mathematics \\ University of California \\ Los Angeles, CA 90095-1555 \\ liu@math.ucla.edu \\ Jie Qing \\ Department of Mathematics \\ University of California \\ Santa Cruz, CA 95064 \\ qing@ cats.ucsc.edu
}

\section{PRODUCTION}

Silvio Levy, Scientific Editor, production@msp.org

\section{SUPPORTING INSTITUTIONS}

ACADEMIA SINICA, TAIPEI

CALIFORNIA INST. OF TECHNOLOGY

INST. DE MATEMÁTICA PURA E APLICADA

KEIO UNIVERSITY

MATH. SCIENCES RESEARCH INSTITUTE

NEW MEXICO STATE UNIV.

OREGON STATE UNIV.

\author{
STANFORD UNIVERSITY \\ UNIV. OF BRITISH COLUMBIA \\ UNIV. OF CALIFORNIA, BERKELEY \\ UNIV. OF CALIFORNIA, DAVIS \\ UNIV. OF CALIFORNIA, LOS ANGELES \\ UNIV. OF CALIFORNIA, RIVERSIDE \\ UNIV. OF CALIFORNIA, SAN DIEGO \\ UNIV. OF CALIF., SANTA BARBARA
}

\author{
Daryl Cooper \\ Department of Mathematics \\ University of California \\ Santa Barbara, CA 93106-3080 \\ cooper@math.ucsb.edu \\ Jiang-Hua Lu \\ Department of Mathematics \\ The University of Hong Kong \\ Pokfulam Rd., Hong Kong \\ jhlu@maths.hku.hk \\ Paul Yang \\ Department of Mathematics \\ Princeton University \\ Princeton NJ 08544-1000 \\ yang@math.princeton.edu
}

These supporting institutions contribute to the cost of publication of this Journal, but they are not owners or publishers and have no responsibility for its contents or policies.

See inside back cover or msp.org/pjm for submission instructions.

The subscription price for 2015 is US \$420/year for the electronic version, and \$570/year for print and electronic.

Subscriptions, requests for back issues and changes of subscribers address should be sent to Pacific Journal of Mathematics, P.O. Box 4163, Berkeley, CA 94704-0163, U.S.A. The Pacific Journal of Mathematics is indexed by Mathematical Reviews, Zentralblatt MATH, PASCAL CNRS Index, Referativnyi Zhurnal, Current Mathematical Publications and Web of Knowledge (Science Citation Index).

The Pacific Journal of Mathematics (ISSN 0030-8730) at the University of California, c/o Department of Mathematics, 798 Evans Hall \#3840, Berkeley, CA 94720-3840, is published twelve times a year. Periodical rate postage paid at Berkeley, CA 94704, and additional mailing offices. POSTMASTER: send address changes to Pacific Journal of Mathematics, P.O. Box 4163, Berkeley, CA 94704-0163.

PJM peer review and production are managed by EditFLOW ${ }^{\circledR}$ from Mathematical Sciences Publishers.

\section{PUBLISHED BY}

\section{mathematical sciences publishers \\ nonprofit scientific publishing}

http://msp.org/

(C) 2015 Mathematical Sciences Publishers 


\section{PACIFIC JOURNAL OF MATHEMATICS}

Volume $276 \quad$ No. $1 \quad$ July 2015

On the degree of certain local $L$-functions

U. K. ANANDAVARdhanan and AmiYa Kumar MondaL

Torus actions and tensor products of intersection cohomology

ASILATA BAPAT

Cyclicity in Dirichlet-type spaces and extremal polynomials II: functions on the bidisk

Catherine Bénéteau, Alberto A. Condori, Constanze Liaw,

DANIEl SECo and Alan A. SOlA

Compactness results for sequences of approximate biharmonic maps

CHRISTINE BREINER and TOBIAS LAMM

Criteria for vanishing of Tor over complete intersections

Olgur Celikbas, Srikanth B. Iyengar, Greg PiePMeyer and

ROGER WIEGAND

Convex solutions to the power-of-mean curvature flow

\section{SHIBING CHEN}

Constructions of periodic minimal surfaces and minimal annuli in $\mathrm{Sol}_{3}$

CHRISTOPHE DESMONTS

Quasi-exceptional domains

ALEXANDRE EREMENKO and ERIK LUNDBERG

Endoscopic transfer for unitary groups and holomorphy of Asai $L$-functions

NEVEN GRBAC and FREYDOON SHAHIDI

Quasiconformal harmonic mappings between Dini-smooth Jordan domains

DAVID KALAJ

Semisimple super Tannakian categories with a small tensor generator

THOMAS KRÄMER and RAINER WEISSAUER

On maximal Lindenstrauss spaces

PETR PETRÁČEK and JiŘí SPURNÝ 